\title{
Log-normal intensity distribution of the quiet-Sun FUV continuum observed by SUMER
}

\author{
J. M. Fontenla ${ }^{1}$, W. Curdt $^{2}$, E. H. Avrett ${ }^{3}$, and J. Harder ${ }^{1}$ \\ ${ }^{1}$ Laboratory for Atmospheric and Space Physics (LASP), University of Colorado, Boulder, CO 80309, USA \\ e-mail: fontenla@lasp.colorado.edu \\ 2 Max-Planck-Institut für Sonnensystemforschung (MPS), 37191 Katlenburg-Lindau, Germany \\ ${ }^{3}$ Harvard-Smithsonian Center for Astrophysics (CfA), 60 Garden Street, Cambridge, MA 02138, USA
}

Received 1 December 2006 / Accepted 21 February 2007

\begin{abstract}
We analyse observations of the quiet-Sun far ultraviolet (FUV) continuum at various wavelengths near $1430 \AA$ obtained by the SUMER instrument on $\mathrm{SOHO}$. According to semi-empirical atmospheric models this continuum originates from the layers in the chromosphere where the temperature rises from low values at near-radiative equilibrium to a plateau of about $6000 \mathrm{~K}$. We study raster images and intensity distribution histograms and find that a single log-normal distribution matches these observations very well, and that the spatial structure observed corresponds to a mixture of features at supergranular and smaller scales that probably correspond to granular clusters. Also, a log-normal distribution was found in the literature to correspond to other chromospheric features and we compare here with histograms obtained from a H I Ly- $\alpha$ quiet-Sun image. Because the continuum around $1430 \AA$ is mainly produced by Si I recombination it is expected to respond well to deep chromospheric heating and not be directly affected by velocities. The data suggest that chromospheric heating originates through dissipation of magnetic free-energy fields of small size and magnitude in underlying photospheric intergranular lanes. It has been suggested that such fields can be produced by photospheric dynamos at the intergranular scale and/or by complex fields emerging in a "magnetic carpet". Such fields are expected to have sufficient free-energy to power the chromospheric heating. Plasma instabilities, such as the Farley-Buneman instability, would allow this free-energy to be dissipated in the chromosphere.
\end{abstract}

Key words. Sun: chromosphere - Sun: magnetic fields

\section{Introduction}

The solar UV continuum in the range around $1400 \AA$ has been observed by several instruments in the past (e.g. ATM/Skylab and UVSP/SMM). However, these instruments were basically designed for studying emission lines and therefore lacked the sensitivity to produce good continuum observations. For instance, data from the UVSP/SMM examined by one of us (Fontenla et al. 1988) had too few counts and therefore was too noisy for the kind of analysis we carry out here. In this paper we examine SUMER observations in the Si I continuum around $1430 \AA$ at several wavelengths where the influence of spectral lines is minimal. Calculations by Vernazza et al. (1981, Fig. 36) indicated that the $1575 \AA$ continuum in their model is formed at the temperature minimum between the solar photosphere and chromosphere, and that the continuum at $1430 \AA$ is formed roughly $200 \mathrm{~km}$ higher, in the low chromosphere, but has almost the same intensity. That model assumed a minimum temperature of $4170 \mathrm{~K}$. In a subsequent revision of the photospheric part of the model given by Maltby et al. (1986, Appendix A) the minimum temperature was increased to $4400 \mathrm{~K}$ in order to improve agreement between the computed and observed $K_{1}$ minimum intensity in the wing of the $\mathrm{Ca}$ II $\mathrm{K}$ line. A recent revision of the low-chromosphere model was carried out by Fontenla et al. (2007) in which it is shown that a low temperature minimum of $3800 \mathrm{~K}$ - roughly consistent with the carbon monoxide (CO) line emission just above the solar limb - is also consistent with the UV continuum in a model with a much steeper temperature rise above the minimum temperature, provided that full-NLTE radiative transfer calculations are carried out. In this model, the temperature minimum is not far from radiative equilibrium (radiative losses are very small) at high gas pressures but soon after the pressure falls below a threshold value (which may be dependent on the magnetic field) the temperature rapidly rises to chromospheric plateau values of $\approx 6000 \mathrm{~K}$ and strong radiative losses occur. In this model the emitted $1430 \AA$ continuum emission mainly originates above the temperature minimum with only a small contribution from the minimum temperature layer. Thus, in this model the Si I continuum emission is a diagnostic of the start of the strong heating in the chromosphere.

Such a rapid change of the radiative losses from very small values to much larger ones indicates a sudden onset of an instability that enables mechanical dissipation of available energy. Fontenla (2007) suggested that the Farley-Buneman instability (hereafter FB instability) is involved in magnetic chromospheric heating of the quiet-Sun where fields of a few Gauss and significant motions are present. The conditions for this mechanism can be expected at those locations where radiative losses become large in the model mentioned above, if such a magnetic field is present.

Several papers, e.g. Henoux \& Somov $(1991,1997)$ have proposed that convective photospheric motions may drive a dynamo effect due to the slippage of neutrals with respect to ions and that this would produce relatively strong fields with important magnetic free-energy. Alternatively, simulations of convection by Sánchez Almeida et al. (2003) have shown that complex 

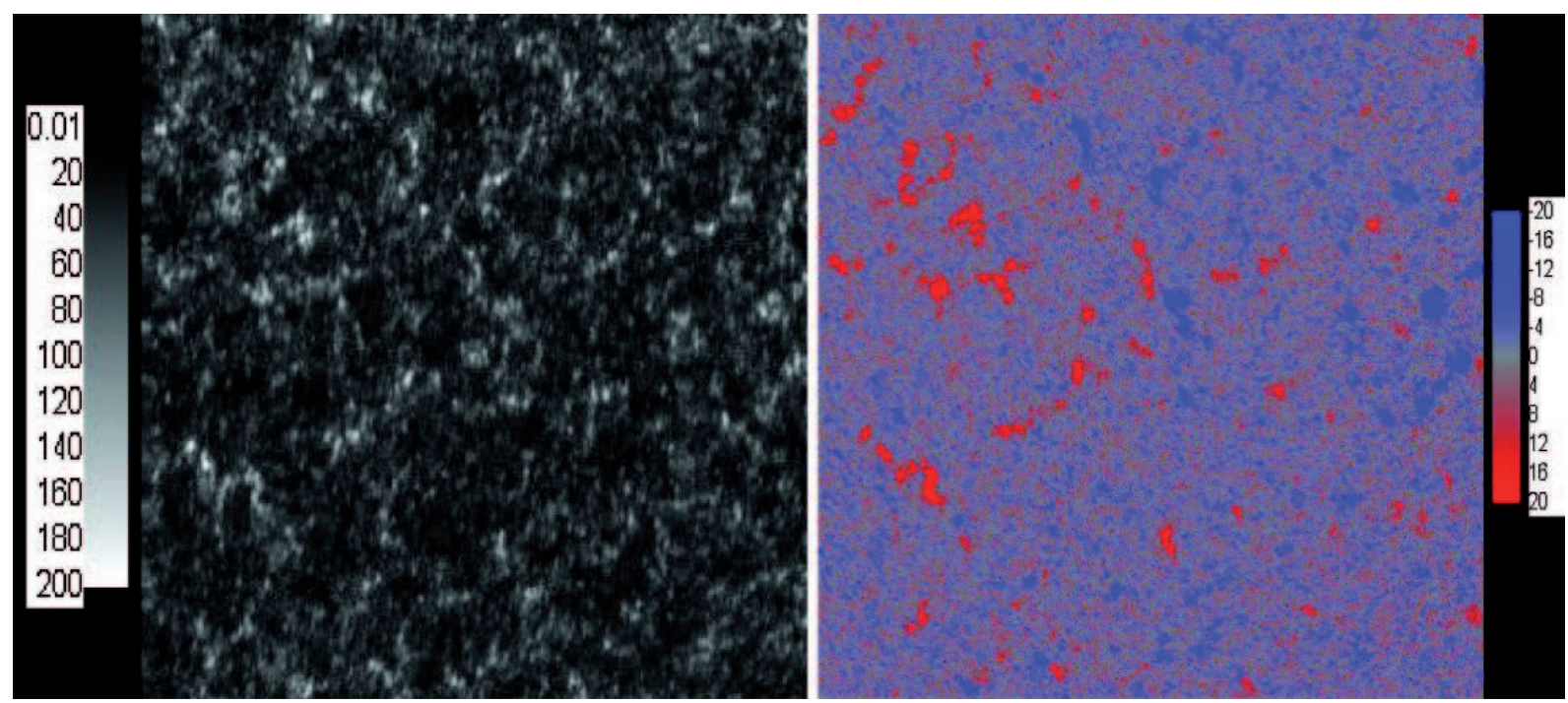

Fig. 1. Left: raster image obtained by SUMER on 1996, Oct. 2 in the continuum at $1430 \AA$ (bandwidth of $2.2 \AA$ ), intensity in $\mathrm{mW} \mathrm{sr}^{-1} \mathrm{~m}^{-2} \AA^{-1}$. Right: MDI magnetogram, flux in Gauss pixel.

magnetic field patterns with important free-energy would result from deeper-seated dynamos. Relatively strong fields at small scales have been observed by various authors, e.g. Parnell (2002, on the so-called "magnetic carpet") and Trujillo Bueno et al. (2004, who indicate a mean magnetic field strength of about 130 Gauss in "turbulent fields"). In the quiet-Sun these fields usually occur at intergranular lanes (Trujillo Bueno et al. 2004) and are stronger at locations where stronger downflows result from several granules. It is not clear at this point how these originate and which are the roles of local dynamos or emergence, but in either case large magnetic free-energy is expected and its effects in heating the chromosphere can be very substantial.

In this paper we show that quiet-Sun chromospheric heating of the base of the chromospheric plateau has a spatial pattern similar to those small-scale magnetic fields and possesses a characteristic log-normal intensity distribution. Also, a lognormal distribution has been observed by Warren et al. (1998) to describe the intensity distribution of features higher in the chromosphere (H I Ly- $\beta$ through Ly-11 and the Ly continuum) and in the transition region. Here we also show that such a type of distribution fits the H I Ly- $\alpha$ intensity distribution observed by the UVSP/SMM instrument (Fontenla et al. 1988), although it has to be modified to fit the Ca II $K_{3}$ data from Meudon Observatory. However, very high-resolution images taken in both of these upper and mid- chromospheric lines show complex loop structures that often connect network lanes with cell interior which are clearly in emission in H I Ly- $\alpha$ (as seen in VAULT data) but sometimes in absorption in Ca II $K_{3}$. The spatial structure of these features is not reflected in the intensity histograms and needs to be further investigated in the context of magnetic fields and energy dissipation. However, because of the limited resolution and sensitivity only the largest of them can be observed and the same is true about the quiet-Sun magnetic fields.

\section{Observations and data reduction}

A raster scan of the quiet Sun was taken by the SUMER instrument on 1996, Oct. 2 from 06:46 to 07:13 UTC with a total duration of 27 minutes. The raster center was at disk center $\left(x=0^{\prime \prime}, y=0^{\prime \prime}\right)$ and the scan was from $x=-150^{\prime \prime}$ to $x=150^{\prime \prime}$ in steps of 0.75 employing detector B and slit $2\left(1^{\prime \prime} \times 300^{\prime \prime}\right)$. We transmitted from each exposure four $2.2 \AA$ wide spectral windows at 1430, 1441, 1444, and $1447 \AA$, which were already onboard flat-field corrected, and spectrally summed up. On the quiet Sun, no prominent spectral line is present in any of these windows (Curdt et al. 2001), neither in the first nor in the second order of diffraction. The onboard activities and telemetry constraints allowed an exposure time of $4 \mathrm{~s}$. The raw data were decompressed and counts converted to spectral radiance in units of $\mathrm{mW} \mathrm{sr}{ }^{-1} \mathrm{~m}^{-2} \AA^{-1}$ using the most recent radiometric calibration procedures. In SUMER spectra, second order contributions will appear superimposed on the first order emission. We have used earlier studies, where the spectral windows were recorded twice, on the $\mathrm{KBr}$-coated and on the bare section of the photocathode, to determine the contribution from the Lyman continuum around $720 \AA$. While this contribution is higher than $50 \%$ at $1600 \AA$ its value is less $5 \%$ at $1440 \AA$ for $\mathrm{KBr}$-recorded spectra. We therefore ignore second order contributions.

A high-resolution magnetogram of the rastered area was obtained by the MDI/SOHO instrument at 07:01 UTC. In hires mode, MDI pixels have a size of $0{ }^{\prime} 605 \times 00^{\prime} 605$ and the exposure time is nominally $0.1 \mathrm{~s}$ (Scherrer et al. 1995). For a pixel-to-pixel comparison of raster and magnetogram the SUMER raster with its angular pixel size of 0 '. $^{\prime} 964$ had to be rebinned. We were able to coalign both images within 1 pixel uncertainty.

\section{Data analysis}

Figure 1 shows a raster image obtained by SUMER on 1996, Oct. 2 that displays small-scale brightening. The brightest elements occur in network lanes, i.e. supergranular cell boundaries, while other brightenings of lower intensity occur at many locations within the cells. Some very faint pixels are also observed. The data for all the continuum bands indicated above have the same pattern, but with some differences in the intensity level and contrast. This confirms that the pattern of bright and dim pixels reflects the observed solar signal that is well above the instrument noise. Also, Fig. 1 shows the part of the MDI magnetogram coaligned with the raster image mentioned above. Despite the fact that the magnetogram is a snapshot but the raster took 27 min to complete, the comparison shows that patches of relatively strong fields of $\approx 30$ Gausss at the network correspond to bright pixels of $\approx 200 \mathrm{~mW} \mathrm{sr}^{-1} \mathrm{~m}^{-2} \AA^{-1}$. Many of the weaker fields also correspond to UV continuum brightness, but 


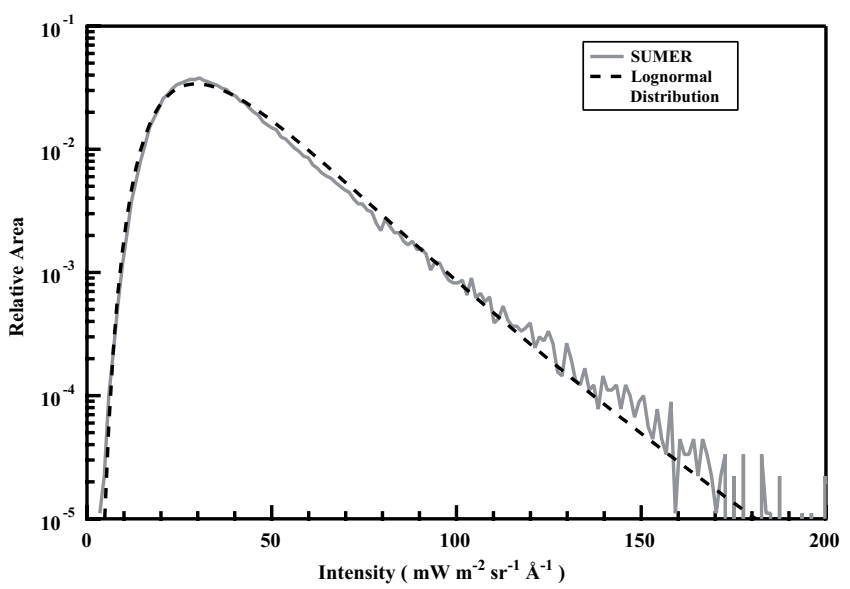

Fig. 2. Intensity histogram of the raster image obtained by SUMER on 1996, Oct. 2 in the continuum at $1430 \AA$ compared with a single lognormal distribution function.

the darker patches at network cell centers would correspond to pixels of only a few Gauss which is too close to the magnetogram noise level. Also the dominance of vertical structures observed in the raster, especially in weaker brightenings, evidently indicates that the raster step cadence is not negligible with respect to the life-time of these events.

In Fig. 2 we show the radiance histogram of the raster depicted in Fig. 1 and compare it with a single log-normal distribution function that follows the following formula,

$f(x)=N \exp \left(\frac{(\ln (x)-\ln (c))^{2}}{2 s^{2}}\right)$

where $N$ is a normalization coefficient, $c$ characterizes the peak location, and $s$ the width of the curve. For the fit in Fig. 2 we used $N=0.034, c=29.5$, and $s=0.564$.

The single log-normal distribution function tracks very well the observed distribution except at the very low intensities where, although SUMER sees few pixels with zero counts, the log-normal yields lower values than observed. An important characteristic of the log-normal distribution is its asymmetric shape and its tail at high values of $x$. These are shared by intensity histograms at many wavelengths where chromospheric emission is dominant. For instance, Fig. 3 shows the intensity histogram from a raster taken by the UVSP instrument on board of SMM on the H I Ly- $\alpha$ line (see Fig. 1 of Fontenla et al. 1988) compared with a log-normal distribution with parameters $N=0.015, c=300, s=0.625$. Here, in contrast to Fig. 2, the observed distribution is below the log-normal at very low intensity values. At large intensity values the observation is very noisy because of the small number of pixels. However, more recent high spatial resolution Ly- $\alpha$ images from the VAULT instrument show that the brightest quiet-Sun features consist of fans of narrow loops mostly originating from points on the supergranular boundaries and extending into the supergranular interior; but also dimmer loops appear in the cell interior and are not apparently connected to the network.

A histogram over a quiet-Sun area near disk center observed at the $\mathrm{Ca}$ II $K_{3}$ line at the Observatoire of Meudon is shown in Fig. 4. Although the shape of the histogram is relatively similar, we find that another parameter is needed in order to obtain a good fit. The function we use is similar to a log-normal but with an offset, as shown in the following equation

$f(x)=N \exp \left(\frac{(\ln (|x-b|)-\ln (c))^{2}}{2 s^{2}}\right)$

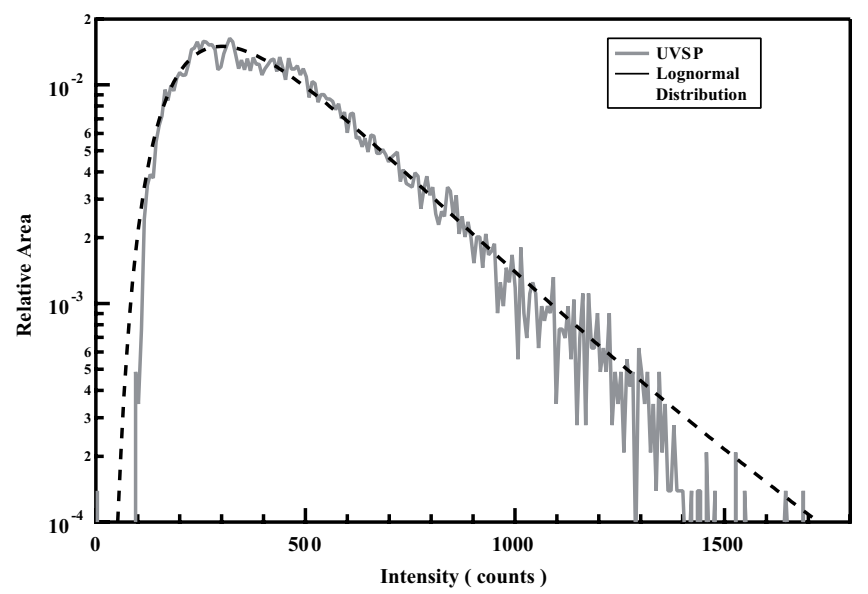

Fig. 3. Intensity histogram of a quiet-Sun raster image obtained by $\mathrm{UVSP} / S M M$ at the H I Ly- $\alpha$ line compared with a single log-normal distribution function.

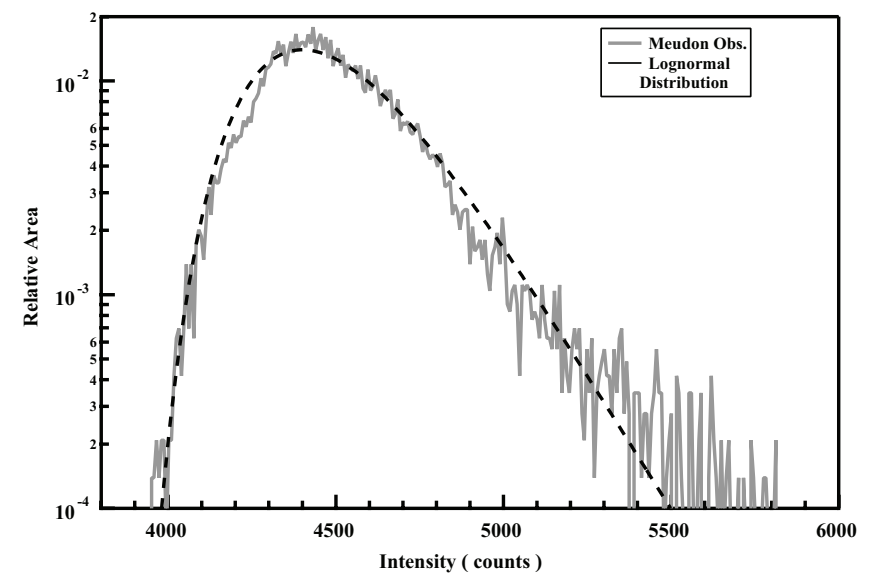

Fig. 4. Intensity histogram of the raster image obtained at the Meudon Observatoire on 2004, Oct. 27 at the Ca II $K_{3}$ line compared with a single modified log-normal distribution function.

where $b$ is the offset. For the fit in Fig. 4 we used $N=0.014$, $b=3720, c=680$, and $s=0.465$. An explanation for the offset is that while the previous spectral features we discussed were mainly pure emission from the chromosphere, the Ca II $K_{3}$ is essentially an absorption line and its intensity is therefore affected by the line scattering of the photospheric background radiation. Intensity distributions for quiet-Sun network cells and lanes have been shown by Harvey \& White (1999, Fig. 7) with a similar distribution to the shown in Fig. 4.

Finally, we show in Fig. 5 a histogram of a quiet-Sun image in the photospheric continuum at around $6060 \AA$ (weak lines are present in the filter range but the continuum dominates) observed by the Precision Solar Photometric Telescope (PSPT, e.g. Vogler et al. 12005; also see http: //www . hao.ucar .edu/ Public/research/fco.html) operated at the Mauna Loa Solar Observatory by the High Altitude Observatory. This histogram shows a very different characteristic in that the asymmetric tail enhancement occurs at low intensities instead of at high intensities. We expect this low intensity tail to arise from unresolved downflows at the intersection of several granules. We find that the distribution is well fitted by a shape similar to a modified log-normal function with an offset $b$, where the log-normal distribution corresponds to intensities above that offset. For the fit in Fig. 5 we used $N=0.011, b=4000, c=545$, and $s=$ 0.15 . A reason to expect a tail for decreasing intensities in the red continuum band is that at this wavelength the observations 


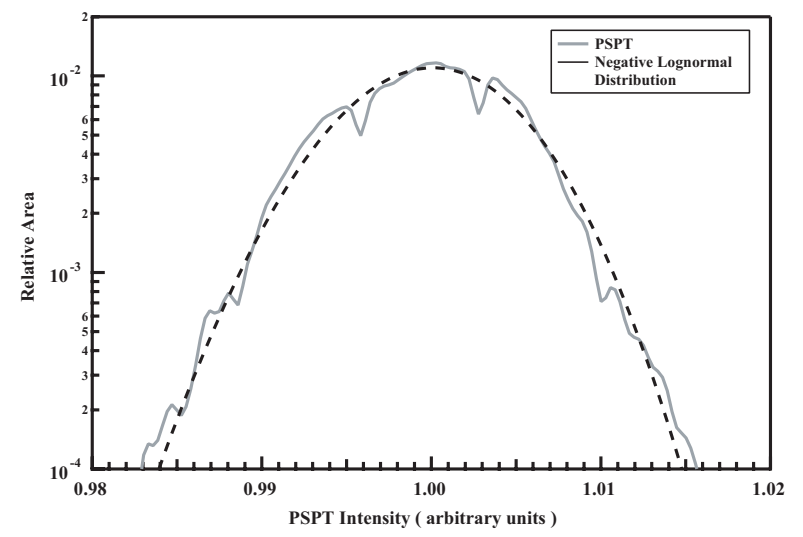

Fig. 5. Intensity histogram of the broadband image obtained at the Mauna Loa Observatory on 2005, Feb. 26 at around $6060 \AA$ compared with a single modified log-normal distribution function. The wiggles are an artifact of digitization and were smoothed.

correspond to unresolved granulation and thus a decreasing intensity is expected at locations of enhanced magnetic fields that correspond to strong convective downflows at the joins of intergranular lanes. However, the shape of the distribution in unresolved images has not yet shown to be predicted from theory.

\section{Discussion}

From the log-normal shape of the quiet-Sun histogram in Fig. 2 we find that the average intensity value of the quiet-Sun atlas by Curdt et al. (2001) is higher than that for the peak (or mode) of the distribution, which corresponds to the value of $c$. This is a characteristics of the log-normal distributions in which the average is given by $\langle x\rangle=c \exp \left(s^{2} / 2\right)$ and thereby is offset from $c$ towards increasing $x$ depending on the width parameter, $s$. The raster image of Fig. 1 shows very substantial intensity variations within the cell center that makes it difficult to define a characteristic value. Thus, the "location" parameter for the distribution, $c=29.5 \mathrm{~mW} \mathrm{sr}^{-1} \mathrm{~m}^{-2} \AA^{-1}$, (which corresponds to $c=\exp (\langle\ln (x)\rangle)$ might be the most logical value to adopt for a cell center model.

Here we have shown that the FUV continuum at around $1430 \AA$ has a log-normal intensity distribution of the same type as is observed in chromospheric emissions of at least H I Ly- $\alpha$, and several other lines and the Ly continuum reported by Warren et al. (1998). These types of distributions are prevalent in many situations in nature, from the IMF to the pollutants in the Earth atmosphere and are expected to reflect a universal property of a certain scaling of physical processes. The continuous shape of the distribution indicates that a similar chromospheric process takes place at the low intensity levels characteristic of network cell centers and in the stronger fields observed in network lanes. The smoothness of the distribution shows that no particular levels of the chromospheric heating rate occur except at the peak of the distribution which is the only local extreme of the log-normal function. Image decomposition procedures based on the addition of a few Gaussian distributions are not meaningful in the context of the observations, but instead a continuous intensity distribution needs to be addressed by a suitable discretization scheme.

Distributions of line-of-sight magnetic flux density are shown by Harvey and White (1999, Fig. 9). These distributions were also studied using MDI/SOHO data by Parnell (2002) who describes the fields as a "magnetic carpet" and indicates a Weibull distribution is an appropriate fit. The Weibull distribution has a tail at high values with a shape similar to that of the log-normal distribution at high values but at low values the magnetic field distribution continues to increase while the log-normal reaches a maximum and then decreases. The similar behaviour at large values suggests a close relationship between the magnetic flux and the chromospheric heating but at low values that relationship breaks down because observational factors might distort the relationship. One possible factor could be noise in the magnetic field measurements that may produce an artificial increase of the area covered by small and noisy values that are not present in the Sun. More recently Domínguez Cerdeña et al. (2006) examined the magnetic field distribution in the quiet-Sun using newer observational methods and found that a log-normal distribution is a good fit to the observations (see, their Fig. 2).

However, there can be physical reasons why there can be differences between the magnetic field distribution and the UV intensity distribution. Chromospheric magnetic heating requires both the presence of a magnetic field that has enough freeenergy, and local plasma conditions that allow that this energy can be dissipated. It is expected that photospheric motions build substantial free-energy when a magnetic field is present and thus, there should not be a chromospheric heating cut-off due to the energy supply. However, there could be a cut-off in the plasma conditions that allow for the local dissipation. In the scenario suggested by Fontenla (2005), the Farley-Buneman instability can only be triggered if the electron magnetization reaches a certain threshold. This condition depends on the magnetic field strength and the plasma density values, and it is possible that very low magnetic fields cannot trigger the dissipation of energy into heating. Conversion of magnetic free-energy to plasma kinetic energy may always occur, but in some cases may be accompanied by heating of the lower chromospheric layers while not in others.

The data we show support a scenario in which small scale photospheric fields of complex topology are responsible for quiet-Sun chromospheric heating. The small-scale fields can be produced by a time-dependent photospheric magnetic dynamo, e.g., similar to the model suggested by Henoux \& Somov (1991, 1997) acting in the converging and down-flowing motions at the edges of the granules. Alternatively, the complex fields may be due to field emergence from a deeper seated dynamo and interaction with the convective motions (cf., simulations by Sánchez Almeida et al. 2003). In any case the magnetic fields should have sufficient free-energy built in from photospheric dynamics to power the chromospheric radiation emission and magneticallydriven motions at small scales. As the gas pressure decreases the velocity increases, but at some point an instability threshold is reached at which anomalous resistivity triggers the production of heat. Such instability may be similar to the scenario proposed by Fontenla (2005) in which the anomalous resistivity and electron heating are due to the FB instability produced by the Hall drift of electrons with respect to ions. Thus, while heating is linked to motions, there is not a close relationship between velocity and UV emission because as energy is dissipated in heating, the energy available for powering faster motions may decrease. Indeed the relationship is dependent on the local conditions and topology that determine how much heating and acceleration occurs. Thus the study of the small-scale magnetic fields in the quiet Sun is a very important tool that when combined with simultaneous UV observations of the region just above the temperature minimum will help to answer some of the fundamental questions relating to the origin and operation of chromospheric heating.

We believe a very likely mechanism that would produce chromospheric magnetic heating with qualitative characteristics 
consistent with observations is the dissipation of magnetic freeenergy by means of the FB instability (described by Farley 1963; and more recently studied by Hamza \& St. Maurice 1995; and Dimant \& Milikh 2003). This mechanism is likely to operate in the conditions of the chromospheric temperature plateau when low ionization plasma is present in a magnetic field and significant electric currents occur perpendicular to the field. In the chromosphere the neutral hydrogen density is larger than the ion density, and the neutral and ion densities become nearly equal only at the top of the chromosphere. The presence of a magnetic field in quiet-Sun regions, where increased heating occurs is shown in Fig. 1 and, as mentioned above, there is substantial evidence for magnetic field structures within the quiet-Sun internetwork, and, of course, for stronger fields in the network. The distribution of the magnetic field is probably related to that of the heating and FUV intensity, but the observations of the brightenings and magnetic fields need both considerable improvement to have better temporal and spatial resolution and lower noise. Statistical relationships between intensity in Ca II and magnetic field were found (e.g., Skumanich et al 1975; Rast 2003; also see Ortiz \& Rast 2005, and references within) but they suffer from the same limitations we have mentioned, and besides, the Ca II intensity is easily affected by velocities, so that these statistical relationships have a large scatter and are not sufficient to definitely establish the physical mechanism by which magnetic heating operates. The FB instability should follow directly as consequence of the conditions mentioned above. The only ingredient that is not currently directly observable is the cross-field electric current. Only extremely high quality vector magnetograms could provide direct information about this. However, from the theoretical standpoint such currents are expected to naturally appear as a result of the dynamics induced by the photospheric motions. These motions have very important components across the field as found in photospheric simulations and are often observed as well. The FB instability is expected because in the conditions of the low chromosphere mostly neutral hydrogen and helium move across magnetic field lines due to gas pressure forces (the plasma $\beta$ is larger than unity) and drive motions of ions and electrons and therefore produce an electric field in the frame of the charged particles, and consequently an electric current. But the ions and electrons are affected by the magnetic field, as described in Fontenla (2005), and the ion collisions with neutrals (mostly charge exchange collisions) are more effective than those of neutrals with electrons in such way that the Hall drift is nearly suppressed for ions but not for electrons (i.e. electrons are highly magnetized but ions are not). The Hall drift of electrons with respect to ions was shown by Farley and Buneman to lead to rapid growth of plasma waves, basically electrostatic ones, and lead to field-aligned irregularities of ionization density. This was found observationally to lead to electron temperature increases (e.g., see Hamza \& St. Maurice 1995; Dimant \& Milikh 2003). These irregularities and electron heating are precisely what is observed in the solar chromosphere, and are likely caused by at least partial dissipation of the cross-field electric-currents by means of the anomalous resistivity resulting from the FB instability. However, a final proof of the operation of this mechanism in the solar chromosphere requires specifically programmed observations of temporal evolution at high spatial resolution and in several spectral lines and continuum together with vector magnetic fields, and comparison of the observations with careful 3D simulation of the MHD and plasma effects (including Hall and Pedersen effects) involved in the FB instability.

Simulation of these effects should include an appropriate treatment not only of MHD but also of non-LTE radiative transfer and losses, ionization, and the plasma instability that dissipates the magnetic free-energy to produce the observed heating. According to this scenario the recipe for a chromosphere is that of convection near the stellar photosphere, generating small-scale magnetic fields, coupled with the conditions for the plasma instability, e.g. plasma with low ionization at sufficiently low gas pressures that the FB instability can occur. We expect that new observations of magnetic fields at much higher resolution and with better signal-to-noise ratio will lead to a better understanding of the relationship between the distributions of the weak fields in the quiet Sun and the chromospheric heating.

The origin of solar UV variability is an important question with implications for atmosphere and climate Earth studies and for solar physics. Studies such as we carry out here will help to interpret the solar irradiance time series observations of the SORCE SOLSTICE instrument (Rottman 2005; Lean et al. 2005). In particular, this will help to understand the role of network and active network as sources of solar UVirradiance variability, and their relationship with magnetic fields at scales smaller than that for active regions; these contributions have been controversial and are potentially important to the UV variability. Only detailed studies, like the one presented here coupled with full Sun modeling, comparison with spatially resolved observations, and spectral irradiance measurements like those been performed by SORCE can provide a definitive answer to these questions.

Acknowledgements. We thank Ingolf Dammasch, who has helped with the data reduction of SUMER spectra. The SUMER project is financially supported by DLR, CNES, NASA and the ESA PRODEX programme (Swiss contribution). SUMER is part of $\mathrm{SOHO}$, the Solar and Heliospheric Observatory, of ESA and NASA. J. M. Fontenla and J. Harder were supported by NASA contract NAS597045 at the University of Colorado.

\section{References}

Curdt, W., Brekke, P., Feldman, U., et al. 2001, A\&A, 375, 591

Dimant, Y. S., \& Milikh, G. M. 2003, GRL, 108, 1350

Domínguez Cerdeña, I., Sánchez Almeida, J, \& Kneer, F. 2006, ApJ, 636, 496

Farley, D. T. Jr. 1963, JGR, 68, 6083

Fontenla, J. M. 2005, A\&A, 442, 1099

Fontenla, J. M, Reichmann, E. J, \& Tandberg-Hanssen, E. 1988, ApJ, 329, 464 Fontenla, J. M., Balasubramaniam, K. S., \& Harder, J. 2007, in Proc. Coimbra Solar Physics Meeting: The Physics of Chromospheric Plasmas, ed. P. Heinzel, I. Dorotovic, \& R.J. Rutten, ASCP Conf. Ser., in press

Hamza, A. M., \& St. Maurice, J.-P. 1995, JGR, 100, 9653

Harvey, K. L., \& White, O. R. 1999, ApJ, 515, 812

Henoux, J. C., \& Somov, B. V. 1991, A\&A, 241, 613

Henoux, J.C., \& Somov, B.V. 1997, A\&A, 318, 947

Lean, J., Rottman, G., Harder, J., \& Kopp, G. 2005, Sol. Phys., 230, 27

Maltby, P., Avrett, E. H., Carlsson, M., et al. 1986, ApJ, 306, 284

Ortiz, A., \& Rast, M. 2005, MmSAI, 76, 1018

Parnell, C. E. 2002, MNRAS, 335, 389

Rast, M. P. 2003, in Local and global helioseismology: the present and future, ed. H. Sawaya-Lacoste, Proc. SOHO 12/GONG+2002, Big Bear Lake, 2002, ESA SP-517, 163

Rottman G. 2005, Sol. Phys., 230, 7

Sánchez Almeida, J., Emonet, T., \& Cattaneo, F. 2003, ApJ, 585, 536

Scherrer P. H., Bogart, P. S., Bush, R. I., et al. 1995, Sol. Phys., 162, 129

Skumanich, A., Smythe, C., \& Frazier, E. N. 1975, ApJ, 200, 747

Trujillo Bueno, J., Shchukina, M., \& Asensio Ramos, A. 2004, Nature, 430, 326 Vernazza, J. E., Avrett, E.H., \& Loeser, R. 1981, ApJS, 45, 635

Vogler, F. L., Brandt, P. N., Otruba, W., \& Hanslmeier, A. 2005, in Proc. 3rd Summerschool and Workshop: Solar Magnetic Phenomena, Solar Observatory Kanzelhöhe, Kärnten, Austria, August 25-September 5, 2003 , ed. A. Hanslmeier, A. Veronig, \& M. Messerotti (The Netherlands, Dordrecht: Springer), Astronomy and Astrophysics Space Science Library, 320, 192

Warren, H. P., Mariska, J. T., \& Wilhelm, K. 1998, ApJS, 119, 105 